\title{
Minimum Mean Square Error Single-User Detection of SDMA Signals Transmitted over Nakagami Fading Channels
}

\author{
Lie-Liang Yang and Lajos Hanzo \\ Dept. of ECS, University of Southampton, SO17 1BJ, UK. \\ Tel: +44-23-8059 3125, Fax: +44-23-8059 4508 \\ Email: 1ly,lh@ecs.soton.ac.uk; http://www-mobile.ecs.soton.ac.uk
}

\begin{abstract}
In this contribution the performance of a spacedivision multiple-access (SDMA) system is investigated, when the space-time block coded signals based on two transmit antennas are transmitted over Nakagami- $m$ fading channels. In the proposed SDMA systems the receiver employs multiple receive antennas and each of the receive antennas consists of several antenna array elements. The antenna arrays' output signals are combined linearly based on the minimum mean square error (MMSE) principles, which only uses the knowledge associated with the desired user, i.e. we consider a single-user MMSE detector. Specific examples are provided for illustrating the properties of the MMSE detector. Our study and simulation results show that the MMSE detector is capable of facilitating joint space-time decoding, beamforming and receiver diversity combining, while simultaneously suppressing the interfering signals.
\end{abstract}

\section{INTRODUCTION}

In wireless communications Multiple-Input-Multiple-Output (MIMO) systems equipped with multiple antennas at both the transmitter and receiver hold the promise of substantial spectral efficiency improvements relative to what is achieved at the current state-of-the-art [1], [2], [3]. Recently, MIMO systems have attracted intensive research interests in the context of both their theory and applications, as indicated by [4], [5] and the references in them. It is widely recognized that MIMO systems can be employed for achieving a high capacity [2], [3], a high diversity order [6], [7], for mitigating various types of interfering signals [8], and for supporting multiple users with the aid of space-division multiple-access (SDMA) [8]. The core principle behind the high spectral efficiency achieved by MIMO systems is that when communicating over a rich scattering propagation environment providing nearly independent transmission paths from each transmit antenna to each receive antenna, the multiple antennas employed by the transmitter and/or receiver are capable of providing extra degrees of freedom in the spatialdomain, in addition to the degrees of freedom provided in the conventionally exploited time-domain and frequency-domain. This increased degrees of freedom allows wireless systems to increase their capacity, to provide increased diversity, or to suppress the effects of interference, etc.

In this contribution the application of the MIMO principle in the context of SDMA is investigated. Specifically, the uplink communication scenario of Fig. 1 is considered, where each mobile user employs two transmit antennas and the base-station (BS) employs $M$ number of receive antenna arrays, each of which invokes $L$ number of array elements or sensors. At the transmitter side Alamouti's space-time coding scheme [6] is employed by the mobiles for encoding the transmitted symbols, in order to achieve transmit diversity gain. By contrast, at the BS the signals obtained by sampling the receive antenna arrays' output are linearly combined, in order to detect the information transmitted by the different SDMA users. In this contribution the employment of single-user detection schemes is studied, where we assume that the receiver has explicit knowledge of the desired user's parameters, including the desired user's direction-of-arrival (DOA) and the corresponding channel impulse responses (CIR) with respect to the $M$ number of receive antenna arrays. However, no knowledge of the other users' parameters is assumed.

The single-user detector considered is the linear minimum mean square error (MMSE) detector [9]. Furthermore, in contrast to the popular Rayleigh fading channel model most often used for studying MIMO systems, in this contribution, the performance of the SDMA system using the above-mentioned MMSE receiver is studied, when communicating over the range of Nakagami- $m$ fading channels. This generic channel model is used, since it often gives the best fit to land-mobile and indoormobile multipath propagation environments, as well as to scintillating ionospheric radio links. Furthermore, the Nakagami- $m$ distribution includes the Rayleigh distribution as a special case for $m=1$. It is also capable of accurately approximating the Rician fading channel associated with high values of $m$, with a one-to-one mapping between the fading parameter $m$ and the Rician $K$-factor.

\section{Representation of The Received Signal}

Fig. 1 shows the structure of a SDMA system, where each mobile transmitter employs two transmit antennas and the common receiver at the base station (BS) employs $M$ number of antenna arrays, each having $L$ elements. Note that in Fig. 1 the thin lines represent scalar variables, while the thick lines correspond to vector variables. As shown in Fig. 1, the adjacent data symbols $x_{1}$ and $x_{2}$ are space-time block coded according to Alamouti's scheme [6] at the $k$ th user's mobile transmitter. The resultant space-time coded symbols are then transmitted during two consecutive symbol periods. In the first symbol period the symbol transmitted from antenna 1 is $x_{k 1}$ and the symbol transmitted from antenna 2 is $x_{k 2}$. By contrast, in the second 
symbol period the symbol transmitted from antenna 1 is $-x_{k 2}^{*}$ and the symbol transmitted from the antenna 2 is $x_{k 1}^{*}$, where the superscript ${ }^{*}$ denotes the complex conjugate.

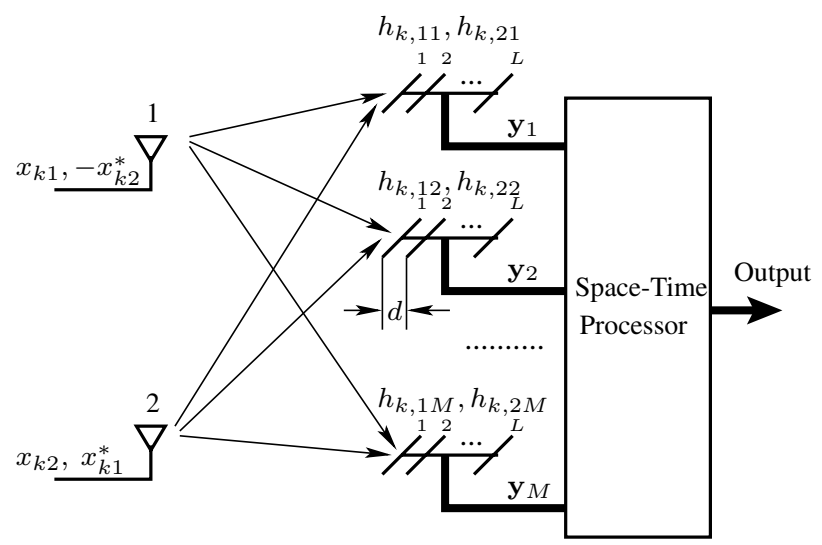

Fig. 1. Stylised schematic of the space-time block coding assisted transmitter, channel model as well as space-time receiver invoked for the SDMA system using two transmit antennas and $M$ receive antenna arrays.

Again, at the base-station there are $M$ number of receiver antenna arrays, as shown in Fig. 1. We assume that all the $M$ different antenna arrays are located sufficiently far apart so that their received signals experience independent fading. As shown in Fig. 1, each of the $M$ antenna arrays consists of $L$ number of array elements, which are linearly correlated elements separated by a distance of $d$. The distance $d$ typically assumes a value of $\frac{\lambda}{2}$, namely half a wavelength. We assume that $K$ users are supported by the SDMA system. These users transmit their data synchronously over flat Nakagami fading channels. Furthermore, we assume that the complex fading envelope remains constant across two consecutive space-time coded symbol periods. Consequently, based on the above assumptions, the received signal vectors of the $m$ th BS antenna array during the two consecutive symbol periods can be expressed as

$$
\mathbf{y}_{m}=\sum_{k=1}^{K} \mathbf{r}_{k m}+\mathbf{n}_{\mathbf{m}}, m=1,2, \ldots, M,
$$

where $\mathbf{y}_{m}$ is a $2 L$-dimensional vector representing the outputs of the $m$ th antenna array associated with a space-time coded period constituted by two consecutive symbol periods, and $\mathbf{n}_{m}$ is a $2 L$-dimensional vector representing the additive white Gaussian noise (AWGN). Each element of $\mathbf{n}_{m}$ is modelled as an independent identically distributed (iid) complex Gaussian random variable with zero mean and a power spectral density of $N_{0} / 2$ per dimension. Furthermore, in (1) $\mathbf{r}_{k m}$ represents the received signal component contributed by the $k$ th user, which can be expressed as

$$
\begin{aligned}
\mathbf{r}_{k m} & =\left[\begin{array}{l}
\mathbf{r}_{k, 1 m} \\
\mathbf{r}_{k, 2 m}^{*}
\end{array}\right] \\
= & {\left[\begin{array}{ll}
\mathbf{d}_{k m} & \mathbf{0} \\
\mathbf{0} & \mathbf{d}_{k m}^{*}
\end{array}\right]\left[\begin{array}{ll}
h_{k, 1 m} & h_{k, 2 m} \\
h_{k, 2 m}^{*} & -h_{k, 1 m}^{*}
\end{array}\right]\left[\begin{array}{l}
x_{k 1} \\
x_{k 2}
\end{array}\right], }
\end{aligned}
$$

where $\mathbf{r}_{k, 1 m}$ and $\mathbf{r}_{k, 2 m}$ represent the $m$ th array's outputs corresponding to the first and second space-time coded symbol periods, respectively, while $\mathbf{d}_{k m}$ represents the $L$-dimensional complex array vector in terms of the $k$ th user and the $m$ th antenna array. Since we assumed that the array elements are separated by half a wavelength, we have

$$
\mathbf{d}_{k m}=\left[1, e^{j \pi \sin \left(\psi_{k m}\right)}, \ldots, e^{j \pi(L-1) \sin \left(\psi_{k m}\right)}\right]^{T},
$$

where $\psi_{k m}$ represents the direction-of-arrival (DOA) corresponding to the $k$ th user and the $m$ th receive antenna array.

In (2) $h_{k, i m}=\left|h_{k, i m}\right| e^{j \theta_{k, i m}}$ represents the channel's impulse response in the context of the $k$ th user, the $i$ th, $i=$ 1,2 , transmit antenna and the $m$ th $m=1,2, \ldots, M$, receive antenna array, where $\left|h_{k, i m}\right|$ represents the fading attenuation, while $\theta_{k, i m}$ represents the phase rotation imposed by the complex-valued Nakagami fading channel. More specifically, we assume that the fading attenuations $\left\{\left|h_{k, i m}\right|\right\}$ are independent Nakagami random variables having a probability density function (PDF) of

$$
p_{\left|h_{k, i m}\right|}(R)=\left(\frac{m}{\Omega}\right)^{m} \frac{2 R^{2 m-1}}{\Gamma(m)} \exp \left(-\frac{m R^{2}}{\Omega}\right),
$$

where $\Gamma(\cdot)$ is the gamma function, $m$ is the Nakagami- $m$ fading parameter, while $\Omega$ in (4) is the second moment of $\left|h_{k, i m}\right|$, i.e., we have $\Omega=E\left[\left|h_{k, i m}\right|^{2}\right]$.

Let

$$
\begin{aligned}
\mathbf{D}_{k m} & =\left[\begin{array}{ll}
\mathbf{d}_{k m} & \mathbf{0} \\
\mathbf{0} & \mathbf{d}_{k m}^{*}
\end{array}\right] \\
\mathbf{H}_{k m} & =\left[\begin{array}{ll}
h_{k, 1 m} & h_{k, 2 m} \\
h_{k, 2 m}^{*} & -h_{k, 1 m}^{*}
\end{array}\right] \text { and } \mathbf{x}_{k}=\left[\begin{array}{l}
x_{k 1} \\
x_{k 2}
\end{array}\right] .
\end{aligned}
$$

Then, $\mathbf{r}_{k m}$ in (2) can be written as

$$
\mathbf{r}_{k m}=\mathbf{D}_{k m} \mathbf{H}_{k m} \mathbf{x}_{k} .
$$

Furthermore, let $\mathbf{Y}=\left[\begin{array}{llll}\mathbf{y}_{1}^{T} & \mathbf{y}_{2}^{T} & \ldots & \mathbf{y}_{M}^{T}\end{array}\right]^{T}$. Then it can be readily shown that the received signal of (1) can be written as

$$
\mathbf{Y}=\sum_{k=1}^{K} \mathbf{D}_{k} \mathbf{H}_{k} \mathbf{x}_{k}+\mathbf{n},
$$

where $\mathbf{D}_{k}$ represents the $k$ th user's array-related matrix, which can be expressed as

$$
\mathbf{D}_{k}=\operatorname{diag}\left(\mathbf{D}_{k 1}, \mathbf{D}_{k 2}, \ldots, \mathbf{D}_{k M}\right), k=1,2, \ldots, K
$$

while $\mathbf{H}_{k}$ is the channel-related matrix of user $k$, which can be expressed as

$$
\mathbf{H}_{k}=\left[\begin{array}{llll}
\mathbf{H}_{k 1}^{T} & \mathbf{H}_{k 2}^{T} & \ldots & \mathbf{H}_{k M}^{T}
\end{array}\right]^{T}, k=1,2, \ldots, K .
$$

Finally, in (7) $\mathbf{n}=\left[\begin{array}{llll}\mathbf{n}_{1}^{T} \mathbf{n}_{2}^{T} & \ldots & \mathbf{n}_{M}^{T}\end{array}\right]^{T}$ is a $2 M L$-dimensional AWGN vector, which is a zero-mean Gaussian vector having a covariance of

$$
\mathbf{R}_{n}=E\left[\mathbf{n n}^{H}\right]=N_{0} \mathbf{I}_{2 M L},
$$


where the superscript ${ }^{H}$ represents the conjugate transpose operation, while $\mathbf{I}_{2 M L}$ is the identity matrix of size $2 M L$.

Having characterized the SDMA signal, below the MMSE detector is investigated, where we assume that the receiver only employs the knowledge of the desired user's parameters. More explicitly, this knowledge includes both that of the array vector in (8) and that of the channel states in (9) in the context of the desired user. Specifically, let us assume that the first user is the user-of-interest, and assume that the receiver employs the knowledge of $\mathbf{D}_{1}$ as well as the knowledge of $\mathbf{H}_{1}$. The objective of the receiver is to detect $\mathbf{x}_{1}=\left[x_{11}, x_{12}\right]^{T}$ transmitted by the user-of-interest associated with the user-index of $k=1$.

\section{MMSE DETECTION OF SDMA SigNALS}

In this section we consider the MMSE detection of the SDMA signals expressed in (7). Firstly, the specific case of supporting $K=2$ users is considered. Then, the results obtained for $K=2$ users are extended to the general case of supporting $K$ number of users.

\section{A. Specific Case of $K=2$ Users}

In the context of the MMSE detector, the received signal vector $\mathbf{Y}$ at the antenna arrays is linearly combined by weighting it with the complex weight matrix $\mathbf{W}$ of size $2 M L \times 2$, in order to yield the estimate $\hat{\mathbf{x}}_{1}$ for the transmitted symbol vector $\mathbf{x}_{1}$ as follows

$$
\hat{\mathbf{x}}_{1}=\mathbf{W}^{H} \mathbf{Y} .
$$

When the MMSE detector is considered, the weight matrix $\mathbf{W}$ is chosen such that the mean-square error between the transmitted symbol vector $\mathbf{x}_{1}$ and the estimated vector $\hat{\mathbf{x}}_{1}$ is minimized, which can be expressed as

$$
\begin{aligned}
\mathbf{W}_{\text {opt }} & =\arg \min _{\mathbf{W}} E\left[\left\|\mathbf{x}_{1}-\hat{\mathbf{x}}_{1}\right\|^{2}\right] \\
& =\arg \min _{\mathbf{W}} E\left[\left\|\mathbf{x}_{1}-\mathbf{W}^{H} \mathbf{Y}\right\|^{2}\right],
\end{aligned}
$$

where the expectation is taken with respect to the symbols and fading amplitudes of the interfering user, as well as the AWGN noise. It can be shown that the solution to the equation of (12) can be expressed as

$$
\mathbf{W}_{o p t}=\mathbf{R}_{y}^{-1} \mathbf{R}_{y x_{1}}
$$

where $\mathbf{R}_{y}$ represents the auto-correlation matrix of $\mathbf{Y}$ given in (7), which is computed by

$$
\mathbf{R}_{y}=E\left[\mathbf{Y} \mathbf{Y}^{H}\right],
$$

while $\mathbf{R}_{y x_{1}}$ is the cross-correlation matrix between the received signal vector $\mathbf{Y}$ of (7) and the desired symbol vector $\mathbf{x}_{1}$, which is given by

$$
\mathbf{R}_{y x_{1}}=E\left[\mathbf{Y} \mathbf{x}_{1}^{H}\right]
$$

Specifically, for the SDMA system supporting two users, $\mathbf{R}_{y}$ of (14) can be expressed as

$$
\begin{array}{r}
\mathbf{R}_{y}=\mathbf{D}_{1} \mathbf{H}_{1} \mathbf{P}_{1} \mathbf{H}_{1}^{H} \mathbf{D}_{1}^{H}+E\left[\mathbf{D}_{2} \mathbf{H}_{2} \mathbf{P}_{2} \mathbf{H}_{2}^{H} \mathbf{D}_{2}^{H}\right] \\
+N_{0} \mathbf{I}_{2 M L},
\end{array}
$$

where $\mathbf{P}_{k}=\operatorname{diag}\left(\left|x_{k 1}\right|^{2},\left|x_{k 2}\right|^{2}\right)$ is related to the transmitted power of user $k, k=1,2$. Note that the expectation in (16) is taken with respect to the antenna array vectors as well as the CIRs of the interfering user. The cross-correlation matrix of (15) can be expressed as

$$
\mathbf{R}_{y x_{1}}=\mathbf{D}_{1} \mathbf{H}_{1} \mathbf{P}_{1} .
$$

The MMSE detector derived above is capable of jointly facilitating space-time decoding, beamforming and receive diversity combining. Below an example is provided for enhancing our understanding of the MMSE detection principles. In this example we assume that the transmitter has two antennas, the BS's receiver has two receive antennas, each of which is equipped with one sensor, and $K=1$ user is supported. In this case, the received vector can be expressed as

$$
\mathbf{Y}=\mathbf{D}_{1} \mathbf{H}_{1} \mathbf{x}_{1}+\mathbf{n},
$$

where $\mathbf{x}_{1}=\left[x_{1} x_{2}\right]^{T}$, while $\mathbf{D}_{1}$ and $\mathbf{H}_{1}$ are given by

$$
\mathbf{D}_{1}=\mathbf{I}_{4}, \mathbf{H}_{1}=\left[\begin{array}{cc}
h_{11} & h_{21} \\
h_{21}^{*} & -h_{11}^{*} \\
h_{12} & h_{22} \\
h_{22}^{*} & -h_{12}^{*}
\end{array}\right] \text {. }
$$

We assume that $\mathbf{P}_{1}=E\left[\mathbf{x}_{1} \mathbf{x}_{1}^{H}\right]=\frac{E_{s}}{2} \mathbf{I}_{2}$, where $E_{s}$ represents the transmitted energy per symbol, while the division by 2 is due to the fact of employing two transmit antennas. Consequently, by following the equations from (11) to (17), we can obtain

$$
\begin{aligned}
\hat{\mathbf{x}}_{1}= & {\left[\left(1+\left(\frac{E_{s}\left(\left|h_{11}\right|^{2}+\left|h_{21}\right|^{2}\right)}{2 N_{0}}\right)^{-1}\right)^{-1}\right.} \\
& \left.+\left(1+\left(\frac{E_{s}\left(\left|h_{12}\right|^{2}+\left|h_{22}\right|^{2}\right)}{2 N_{0}}\right)^{-1}\right)^{-1}\right] \mathbf{x}_{1} \\
& +\mathbf{R}_{y x}^{H} \mathbf{R}^{-H} \mathbf{n},
\end{aligned}
$$

which shows that the received signal has been successfully space-time decoded and, simultaneously, both transmit as well as receive diversity gains were achieved, as indicated by the terms of $\left|h_{11}\right|^{2}+\left|h_{21}\right|^{2}$ and $\left|h_{12}\right|^{2}+\left|h_{22}\right|^{2}$.

\section{B. General Case of K Users}

The philosophy of the simple MMSE detectors derived in the above subsection can be readily extended to the general case of considering SDMA systems supporting $K$ number of users. The corresponding estimate of the transmitted symbols of user 
1 is also given by (11), where the optimum weight vector is given by (13). The auto-correlation matrix of the received signal vector $\mathbf{Y}$ is now given by

$$
\begin{aligned}
\mathbf{R}_{y}= & E\left[\left(\sum_{k=1}^{K} \mathbf{D}_{k} \mathbf{H}_{k} \mathbf{x}_{k}+\mathbf{n}\right)\left(\sum_{k=1}^{K} \mathbf{D}_{k} \mathbf{H}_{k} \mathbf{x}_{k}+\mathbf{n}\right)^{H}\right] \\
= & \mathbf{D}_{1} \mathbf{H}_{1} \mathbf{P}_{1} \mathbf{H}_{1}^{H} \mathbf{D}_{1}^{H}+\sum_{k=2}^{K} E\left[\mathbf{D}_{k} \mathbf{H}_{k} \mathbf{P}_{k} \mathbf{H}_{k}^{H} \mathbf{D}_{k}^{H}\right] \\
& +N_{0} \mathbf{I}_{2 M L},
\end{aligned}
$$

where $\mathbf{P}_{k}=\operatorname{diag}\left(\left|x_{k 1}\right|^{2},\left|x_{k 2}\right|^{2}\right), k=1, \ldots, K$ is related to the transmitted power of the $k$ th user. The cross-correlation matrix evaluated for the received signal vector $\mathbf{Y}$ and the desired symbol vector $\mathbf{x}_{1}$ can be derived as

$$
\begin{aligned}
\mathbf{R}_{y x_{1}} & =E\left[\left(\sum_{k=1}^{K} \mathbf{D}_{k} \mathbf{H}_{k} \mathbf{x}_{k}+\mathbf{n}\right) \mathbf{x}_{1}^{H}\right] \\
& =\mathbf{D}_{1} \mathbf{H}_{1} \mathbf{P}_{1},
\end{aligned}
$$

which is independent of the interfering users.

Having characterised the MMSE detector analytically, let us now provide a range of numerical results in the following section.

\section{Performance Results}

In this section we provide a range of simulation results, in order to illustrate the achievable performance of the SDMA system considered in conjunction with the MMSE receiver. As an example, we assumed that each SDMA mobile employed two transmit antennas, while the $\mathrm{BS}$ employed $M=2$ receive antenna arrays, each having $L=2$ array elements. Furthermore, in order to provide an insight into the effects of the multiuser interference on the users' spatial signature, we assumed that only $K=2$ users were supported by the SDMA system, where one of them was the desired user and the other one was the interfering user.

Fig. 2 shows the bit error rate (BER) versus signal-to-noise (SNR) per bit $\left(E_{b} / N_{0}\right)$ performance of the SDMA system, with respect to the Nakagami fading parameters of $m=$ $0.5,0.8,1.0,1.2,1.5,1.8$ and 2.0. In Fig. 2 we assumed that the SDMA system supported just $K=1$ user, hence no multiuser interference was imposed on the desired user. As shown in Fig. 2, the BER performance improves, as the fading becomes more benign, i.e. as the fading parameter's value increases. According to the results of Fig. 2 we observe that in the absence of multiuser interference the MIMO based SDMA system is capable of achieving an impressively low BER value in the low SNR per bit range. As shown in Fig. 2, in the worst considered transmission environment, which corresponded to $m=0.5$, a BER of $10^{-3}$ can be achieved at an SNR of $E_{b} / N_{0}=8 \mathrm{~dB}$. By contrast, for the best case considered namely in the context of $m=2.0$, a BER of $10^{-5}$ may be achieved at an $\mathrm{SNR}$ of $E_{b} / N_{0}=6.5 \mathrm{~dB}$. Note that such a low BER is achieved,

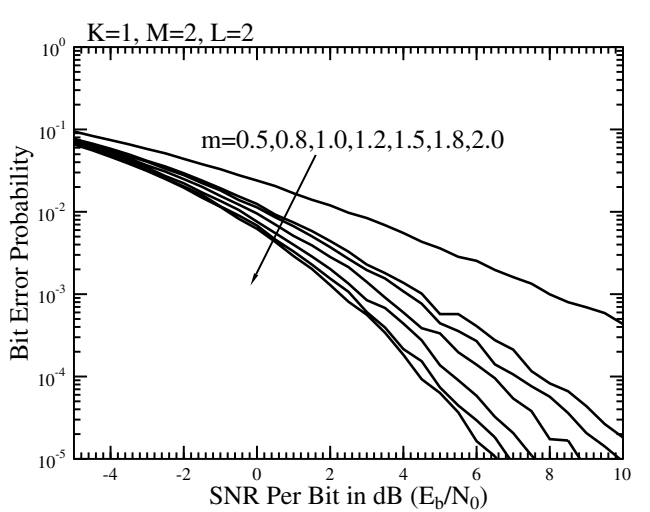

Fig. 2. BER versus SNR per bit performance of the SDMA system using $T x=2$ transmit antennas, $M=2$ receive antenna arrays, each having $L=2$ sensors, and supporting $K=1$ user, when communicating over the Nakagami- $m$ fading channels corresponding to the fading parameters of $m=0.5,0.8,1,1.2,1.5,1.8,2.0$.

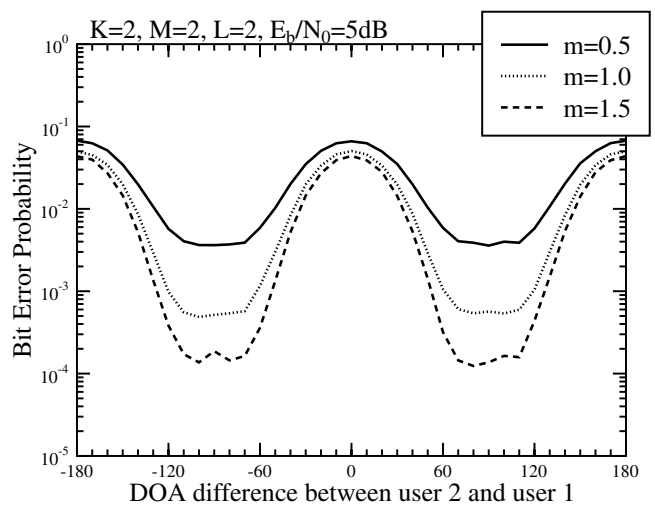

Fig. 3. BER performance of the SDMA system with respect to different direction-of-arrival (DOA) for the interfering signals. In our simulations, we assumed that, the DOAs with respect to the first and second BS receive antennas of the desired user were fully correlated, i.e. we assumed that we had $\psi_{11}=\psi_{12}=0^{\circ}$ and $\psi_{21}=\psi_{22}$. The DOA difference is defined as $\psi_{21}-\psi_{11}$. The other parameters used were $T_{x}=2, M=2, L=2$ and the Nakagami- $m$ fading parameters were $m=0.5,1,1.5$.

since the SDMA system is capable of efficiently amalgamating the benefits of transmit diversity, receive diversity as well as receiver beamforming.

In order to demonstrate the effects of different DOAs on the achievable BER performance, Fig. 3, Fig. 4 and Fig. 5 show the BER versus the DOA performance of the SDMA system considered. In the context of Fig. 3 we assumed that the DOA of the rays arriving from a mobile user to the $M$ different $\mathrm{BS}$ receive antenna arrays are fully correlated. More explicitly, we assumed that for the desired user we had $\psi_{11}=\psi_{12}=0^{\circ}$, while for the interfering user we had $\psi_{21}=\psi_{22}$ associated with equiprobable values in the range of $\left[-180^{\circ}, 180^{\circ}\right]$. In the con- 
text of Fig. 4 and Fig. 5 we assumed that the DOAs of each of the transmit antennas with respect to each of the receive antenna arrays were independent. The results of Fig. 4 and Fig. 5 were obtained by assuming that we had $\psi_{11}=\psi_{12}=0^{\circ}$, and that both $\psi_{21}$ and $\psi_{22}$ assumed random, equiprobable values in the range of $\left[-180^{\circ}, 180^{\circ}\right]$. Furthermore, the results of Fig. 4 were generated, when communicating over a Rayleigh fading channel associated with $m=1$, while those of Fig. 5 for Nakagami$m$ fading channels associated with $m=1.5$. From the results of Figs. 3, 4 and Fig. 5 we observe that when the interfering signals arrive from a sufficiently different DOA, the multiuser interference can be substantially mitigated by the receiver with the aid of the proposed MMSE processing of the array signals..: Furthermore, the results of Fig. 4 and Fig. 5 show that the BER performance can be significantly improved, provided that the interfering signal is orthogonal to the desired signal at either of the receive antenna arrays. However, when the interfering signals have DOAs similar to those of the desired signals at both of the receive antenna arrays, then the interfering user will impose strong interference on the desired users. Furthermore, by comparing 4 and Fig. 5 we can see that when the channel quality improves, i.e. when the value of $m=1.0$ used in Fig. 4 is increased to $m=1.5$ in Fig. 5, the achievable BER performance also improves.

Note that in our simulations portrayed in Figs. 3, 4 and Fig. 5 we assumed that each of the $M$ receive antenna arrays employed $L=2$ array elements. If each receive antenna array has a high number of array elements, then the antenna arrays are capable of achieving a high DOA resolution.

In summary, our simulation results show that the proposed SDMA system is capable of suppressing the multiuser interfering signals having a DOA sufficiently different from that of the desired signals. However, without the knowledge of the interfering signals, strong interference may be imposed on the desired signals, when the multiuser interfering signals are received from directions similar to those of the desired signals.

\section{REFERENCES}

[1] J. H. Winters, "On the capacity of radio communication systems with diversity in a Rayleigh fading environment," IEEE Journal on Selected Areas in Communications, vol. SAC-5, pp. 871-878, June 1987.

[2] I. E. Telatar, "Capacity of multiantenna Gaussian channels," European Trans. on Telecommun., vol. 10, pp. 585-595, Nov./Dec. 1999.

[3] A. Goldsmith, S. A. Jafar, N. Jindal, and S. Vishwanath, "Capacity limits of MIMO channels," IEEE J. on Select. Areas in Commun., vol. 21, pp. 684702, June 2003.

[4] "Special issue on MIMO systems and applications," IEEE Journal on Selected Areas in Communications, vol. 21, April 2003.

[5] "Special issue on MIMO systems and applications," IEEE Journal on Selected Areas in Communications, vol. 21, May 2003.

[6] S. M. Alamouti, "A simple transmit diversity technique for wireless communications," IEEE Journal on Selected Areas in Communications, vol. 16, pp. 1451-1458, October 1998.

[7] V. Tarokh, N. Seshadri, and A. R. Calderbank, "Space-time codes for high data rate wireless communication: performance criterion and code construction," IEEE Transactions on Information Theory, vol. 44, pp. 744765, March 1998.

[8] A. F. Naguib and R. Calderbank, "Space-time coding and signal processing for high data rate wireless communications," Wireless Communications and Mobile Computing, vol. 1, pp. 13-34, January-March 2001.

[9] S. Verdu, Multiuser Detection. Cambridge University Press, 1998.

$$
K=2, M=2, L=2, m=1.0, E_{b} / N_{0}=5 d B
$$

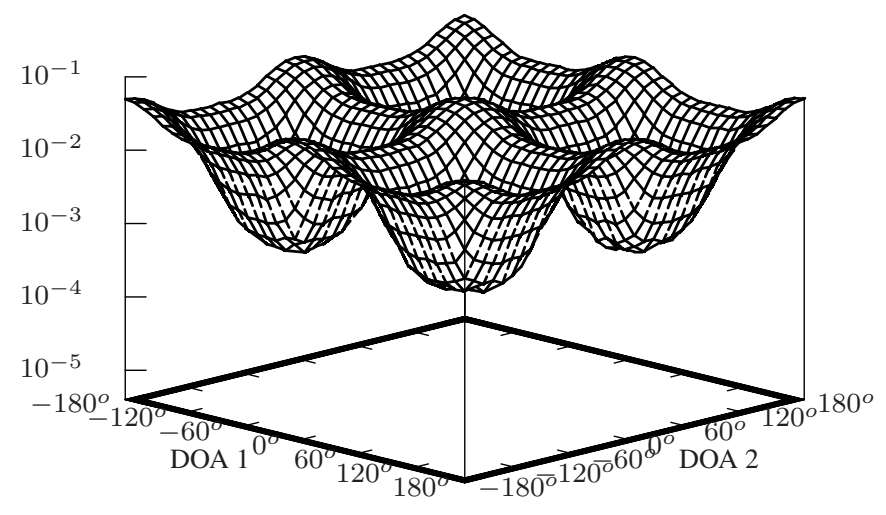

Fig. 4. BER versus DOA performance of the SDMA system using $T x=2$ transmit antennas, $M=2$ receive antenna arrays each having $L=2$ sensors, and supporting $K=2$ users, when communicating over Rayleigh fading channels corresponding to $m=1$. In this figure DOA 1 and DOA 2 represent the DOA differences between the signals received from user 2 and user 1 , by the first and second antenna arrays respectively.

$$
K=2, M=2, L=2, m=1.5, E_{b} / N_{0}=5 d B
$$

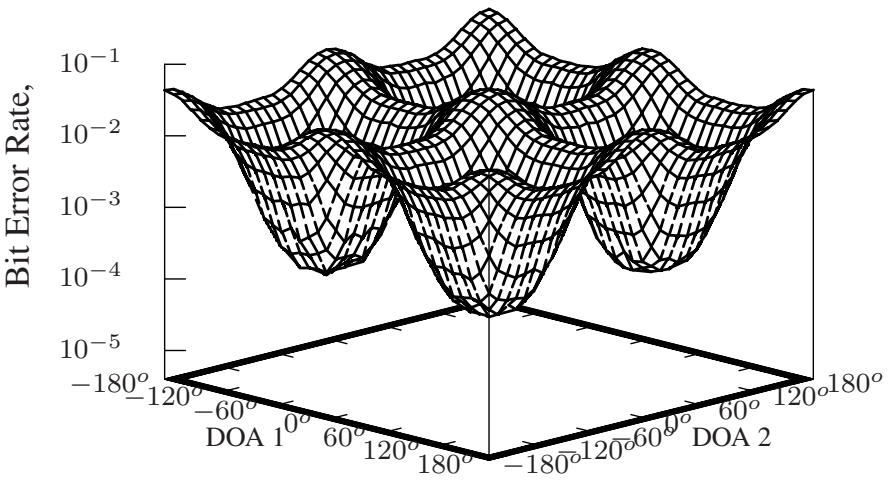

Fig. 5. BER versus DOAs performance of the SDMA system using $T x=2$ transmit antennas, $M=2$ receive antenna arrays each having $L=2$ sensors, and supporting $K=2$ users, when communicating over Nakagami fading channels corresponding to $m=1.5$. In this figure DOA 1 and DOA 2 represent the DOA differences between the signals received from user 1 and user 2, by the first and second antenna arrays respectively. 